
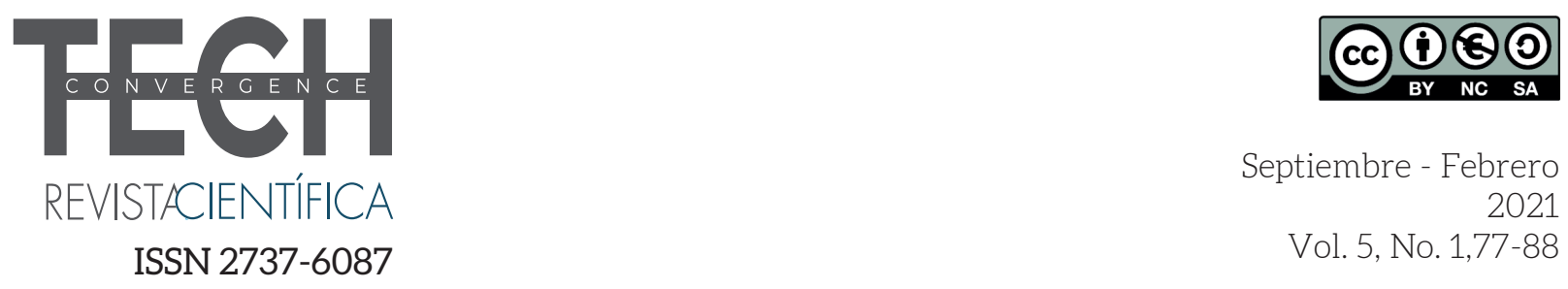

Septiembre - Febrero

2021

Vol. 5, No. 1,77-88

\title{
USO DE LAS MEMORIAS TÉCNICAS EN LA ELABORACIÓN DE PRODUCTOS QUE RESCATAN LA SIMBOLOGÍA CAÑARI
}

\author{
Estudiantes del 1er ciclo de la Carrera de Diseño Gráfico* \\ PhD. María Teresa Pantoja Sánchez ${ }^{1}$ \\ Vicerrectora del Instituto Superior Tecnológico Particular Sudamericano, Cuenca \\ E-mail: mtpantoja@sudamericano.edu.ec \\ (iD) https://orcid.org/0000-0002-3241-6241
}

\section{RESUMEN}

Con un avance a una velocidad incontrolable de la tecnología y el interés generado en las nuevas generaciones en consumir productos provenientes de otras culturas totalmente diferentes a ecuatoriana, se ha desvalorizado y sobre todo se ha relegado a un segundo plano todo el bagaje de conocimientos, tradiciones, costumbres que representan al Ecuador tal, lo cual demuestra el verdadero origen y la riqueza cultural del país; por ello gracias a este proyecto ha surgido la idea de rescatar estos valores que se han perdido, haciendo un replanteamiento de la inmensa simbología Cañari para representarla un nuevo diseño que aún contenga las características morfológicas de los signos Cañari. El objetivo general de la investigación consistió en aplicar las memorias técnicas en el rescate y revalorización de la simbología Cañari por medio de la construcción de un nuevo módulo que conserve las partes morfológicas y tipológicas de su diseño original, aplicado en tazas de cerámica. A nivel metodológico, la investigación parte de un análisis documental de las principales referencias bibliográficas sobre la simbología Cañari y su importancia histórica en la en la consolidación cultural del Ecuador. La aplicación de un cuestionario mediante el uso de un software libre permitió el diseño del bocetaje basada en el análisis de la información obtenida de la opinión de expertos en la simbología Cañari hasta llegar a un módulo óptimo. El desarrollo de la propuesta desde los conocimientos de las técnicas del Diseño Gráfico es un aporte fundamental para el futuro desempeño como tecnólogo para resolver problemáticas mediante la propuesta de un proyecto, como alternativa de solución. De igual manera, incluyendo los conocimientos técnicos y teóricos de cada una de las asignaturas, se desarrollarán las habilidades, procedimientos y conocimientos propios del campo de la investigación, los cuales pueden llegar a formar parte del cúmulo tecnológico de la institución, para dar respuesta a las necesidades de la sociedad.

Palabras Clave: simbología Cañari, memorias técnicas, diseño gráfico

\section{ABSTRACT}

With an uncontrollable advance of technology and the interest generated in the new generations in consuming products from other cul- tures totally different from Ecuador, the whole baggage of knowledge, traditions has been devalued and above all has been relegated to the background. , customs that represent such Ecuador, which shows the true origin and cul- 
tural richness of the country; For this reason, thanks to this project, the idea of rescuing these values that have been lost has arisen, making a rethinking of the immense Cañari symbolism to represent a new design that still contains the morphological characteristics of the Cañari signs. The general objective of the research was to apply the technical memories in the rescue and revaluation of the Cañari symbolism through the construction of a new module that preserves the morphological and typological parts of its original design, applied to ceramic mugs. At the methodological level, the research starts from a documentary analysis of the main bibliographic references on the Cañari symbolism and its historical importance in the cultural consolidation of Ecuador. The application of a questionnaire through the use of free soft- ware allowed the design of the sketch based on the analysis of the information obtained from the opinion of experts in Cañari symbology until reaching an optimal module. The development of the proposal from the knowledge of the techniques of Graphic Design is a fundamental contribution for the future performance as a technologist to solve problems by proposing a project, as an alternative solution. In the same way, including the technical and theoretical knowledge of each of the subjects, the skills, procedures and knowledge of the research field will be developed, which can become part of the technological accumulation of the institution, to respond to the needs of society.

Keywords: Cañari symbols, technical reports, graphic design

Forma sugerida de citar: Pantoja Sánchez, M. T. (2021). Uso de las memorias técnicas en la elaboración de productos que rescatan la simbología Cañari. Convergence Tech Revista Científica. 5(1), 77-88. https://doi.org/10.53592/convtech.v5iV.36

\section{INTRODUCCIÓN}

A continuación, se presentan los resultados del Proyecto Integrador de Saberes desarrollado por estudiantes de la Carrera Diseño Gráfico del Instituto Superior Tecnológico Particular Sudamericano con el objetivo de promover el rescate de los signos y símbolos de la cultura Cañari, así como la revalorización de los mismos. Como una forma de apoyar a las constantes ferias artesanales que acontecen a lo largo y ancho de país, que tiene como finalidad el auto sustentarse como artesanos a la vez que damos a conocer al mundo nuestro valor cultural.

Estas ferias son una pequeña demostración de la riqueza cultural del Ecuador, sin embargo, se ha hecho más frecuente entre los cuencanos el adoptar modas que pertenecen a otras culturas, como es el caso de la gran demanda que tienen los productos importados, además de un gran número de artículos para el hogar. En este sentido, el tema planteado para el proyecto integrador de saberes es la utilización de las memorias técnicas en el desarrollo tasas decorativas que rescaten la simbología de la Cultura Cañari, siendo congruentes al responder al eje integrador de saberes del primer ciclo,
Memorias Técnicas y al uso de las técnicas propuestas por la Coordinación de la Carrera de Tecnología en Diseño Gráfico.

Para empezar, se inició con el planteamiento del problema, luego el objetivo general, los objetivos específicos, marco de referencia y marco teórico. En este último apartado, se abordan los diferentes hechos históricos de la cultura Canarí; desde su origen, cultura, religión, gastronomía, costumbres y festividades. Dada la naturaleza del tema se desarrolló un marco conceptual, en el cual se incluyen todos los conceptos relevantes para el proyecto. Posteriormente se realizó una investigación descriptiva, con un enfoque cuantitativo, para recabar la información, se diseñó una encuesta, la cual se aplicó en línea haciendo uso de software libre. Posteriormente, se realizó un análisis de la información, en base a los resultados obtenidos partimos con las propuestas, y, por último, se elaboraron las conclusiones, recomendaciones, bibliografía y anexos.

Con la elaboración del proyecto pretende sensibilizar a la población respecto al consumo de productos elaborados con la simbología de nuestra Cultura Cañari, de esta forma, lograremos, fortalecer la identidad nacional. 
La principal motivación para llevar a cabo nuestro proyecto es el de rescatar la simbología Cañari, rescatando su cultura, costumbres, tradiciones, festividades... características que se pierden con la constante globalización y expansión de culturas extranjeras. Rescatar nuestra cultura mediante un proyecto integrador de saberes, nos permite como futuros profesionales, integrar en un solo proyecto todos los conocimientos adquiridos durante el ciclo cursado, además que nos prepara mediante la utilización de las memorias técnicas, para el ejercicio profesional del tecnólogo en diseño, el cual utilizará como herramienta de trabajo las memorias técnicas, las mismas que permiten desarrollar una metodología de trabajo, muy similar a la propuesta en un proyecto.

La elaboración de un proyecto nos prepara para la práctica de la profesión, al tener que resolver una problemática, mediante la propuesta de un proyecto, como alternativa de solución. Además, al ir incluyendo los conocimientos técnicos y teóricos de cada una de las asignaturas, vamos desarrollando; habilidades, procedimientos y conocimientos propios del campo de la investigación, los cuales pueden llegar a formar parte del cúmulo tecnológico de la institución, para dar respuesta a las necesidades de la sociedad. Para la realización del mismo contamos con todos los conocimientos teóricos y prácticos, adquiridos durante nuestra formación, los cuales están establecidos en la malla curricular de las carreras que se ofertan en la institución.

\section{MÉTODOS}

El método de investigación, nos señala la forma en cómo se recabará la información, mediante los datos obtenidos, esto nos permite hacer un análisis e interpretación de los resultados. El paradigma de investigación en el que se inscribe el presente proyecto de investigación es el interpretativo, con un enfoque cualitativo. Se realizó una investigación descriptiva, con el propósito de recabar la información, se diseñó una encuesta, con preguntas cerradas y abiertas, la cual se aplicó utilizando el muestreo aleatorio simple. Se aplicó una encuesta de opinión, por lo que se efectuó en línea, se notificó a un grupo pequeño de conocidos (amigos y familiares) que se había subido una encuesta a una plataforma, se dejó durante un periodo comprendido entre tres días y una semana. La elaboración del cuestionario fue mediante el uso de un software libre. Posteriormente, se hizo un análisis de la información, esto nos permitió poder diseñar la propuesta, en base a los resultados obtenidos en la encuesta de opinión.

A continuación, se presentan los resultados obtenidos y el análisis de la información, se puede consultar el cuestionado aplicado en el anexo 1.

\section{PROPUESTA DEL PROYECTO}

Para la elaboración de la propuesta, se realizó un análisis comparativo entre Las Memorias Técnicas y el PIENSA.

\begin{tabular}{ll}
\hline \multicolumn{1}{c}{ MEMORIAS TÉCNICAS } & \multicolumn{1}{c}{ APLICACIÓN EN EL PIENSA } \\
\hline Título del proyecto & Título del proyecto \\
\hline Objetivos & Objetivos \\
\hline $\begin{array}{l}\text { Composición del equipo } \\
\text { investigador }\end{array}$ & Autores \\
\hline Áreas de Investigación & Eje integrador de saberes del PIENSA \\
\hline Área del conocimiento & $\begin{array}{l}\text { Todas las asignaturas del ciclo, descripción breve de la } \\
\text { asignatura }\end{array}$ \\
\hline
\end{tabular}




\begin{tabular}{cc}
\hline MEMORIAS TÉCNICAS & APLICACIÓN EN EL PIENSA \\
\hline $\begin{array}{c}\text { Fundamento del estudio y } \\
\text { significado }\end{array}$ & Justificación \\
\hline Métodos & Metodología \\
\hline Desarrollo del proyecto & Propuesta del proyecto \\
\hline Plan de difusión de los resultados & Presentación del PIENSA en la casa abierta y evaluación del PIENSA \\
& en las aulas. \\
\hline Presupuesto & Presupuesto \\
\hline Requerimientos & La descripción de la aplicación de cada una de las asignaturas. \\
\hline Anexos & Anexos \\
\hline
\end{tabular}

A continuación, se presenta, la descripción de cada una de las asignaturas que se cursaron en el primer ciclo, la aplicación de cada una de ellas en el proyecto y las evidencias correspondientes.

Descripción breve del arte, cultura y diseño nacional: Se abordan los diferentes hechos históricos de la cultura Canarí; desde su origen, cultura, religión, gastronomía, costumbres y festividades.

Descripción de la aplicación: El proceso histórico abordado en la asignatura nos ha ayudado para fomentar las bases de nuestro proyecto, ya que, con los conocimientos históricos de la cultura Cañari, partimos con la información necesaria para elaborar un proyecto que pueda ser defendido en la entrega posterior.

Descripción breve de la representación gráfica: Esta asignatura aportó la técnica y el conocimiento para desarrollar la parte gráfica, la cual se ve representada en: los bocetajes y el diseño del producto.

Descripción de la aplicación: En el proceso de elaboración del símbolo, que rescata la cultura Cañari, se aplicarán los diferentes conocimientos teóricos y técnicas aprendidas en las horas clase, para definir de una forma correcta el diseño. Se aplicará la parte de sombreado, trazos, líneas, luces y sombras, también el uso correcto de formas orgánicas.

Descripción breve de la asignatura teoría del diseño: La teoría del diseño abarca la correcta reestructuración de los diferentes signos y símbolos, es la que se colocará adecuadamente en el marco del diseño del proyecto.
Descripción de la aplicación: Con los conocimientos adquiridos en la asignatura, estamos en óptimas condiciones de seleccionar ciertos signos Cañari, luego de ello se procederá, al análisis tanto morfológico como tipológico, en dicho proceso se recaudará información la cual utilizaremos para realizar nuestro diseño.

Descripción breve de semiología y color: Los conocimientos adquiridos dentro de esta asignatura, abarcan la teoría del color, también observamos los diferentes tipos de contraste, como, por ejemplo: contraste simultáneo, complementarios, luminosidad, temperatura, saturación, valor, entre otros.

Descripción de la aplicación: La cromática que se va a utilizar, para el proyecto, va a ser tomada en su mayoría de las diferentes aplicaciones en textiles, principalmente

Descripción breve de comunicación oral y escrita: Se identifican las diferentes formas de leer y de cómo estas nos facilitan la forma en la que entendemos una lectura, además de ello nos brinda la información de cómo llegar a nuestro espectador de una manera concisa y sobre todo que el espectador entienda nuestro mensaje a transmitir, para ellos estudiamos los diferentes elementos del proceso comunicativo.

Descripción de la aplicación: Se elabora el proyecto escrito para el cumplimiento de las diferentes normativas que se especifican en las memorias técnicas. También nos permite expresar nuestras ideas y diferentes procesos vividos, durante la elaboración de nuestro proyecto. Nos prepara para el momento de exponer nuestro proyecto ante los docentes. 
Descripción breve del proyecto integrador de saberes: memorias técnicas: La facilitadora o guiadora de todo este proyecto, ya que la maestra es la que nos ayuda a dirigir de cierta el timón de nuestro barco, es decir que nos ayuda a avanzar en el transcurso de elaboración de nuestro producto, Enfocándonos en la parte escrita y estructuración.

Descripción de la aplicación: Nos dirige de manera adecuada y ordenada el rumbo de nuestro proyecto integrador, dando explicaciones de acuerdo a nuestros avances, además de realizar el seguimiento a cada proyecto.

\section{RESULTADOS}

A continuación, se presentan los productos obtenidos.

\section{TAZAS}

TRABAJO DE: Franklin Ronaldo Guapacasa Yanza
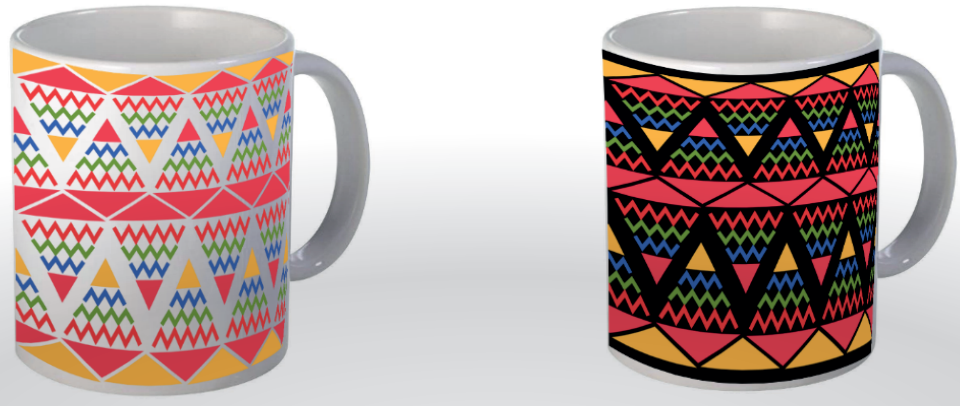

\section{CHOMPAS}

TRABAJO DE: Eduardo Benavides, Paúl Zhunio, Lauro Calle.
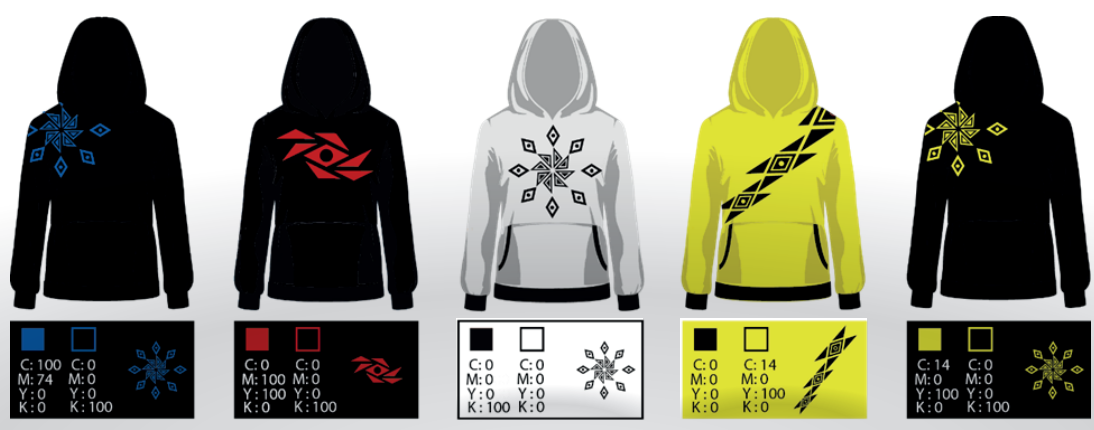

\section{MANTELES}

TRABAJO DE: Melina Pesantez Paulina Urgilés, Sebastián Arévalo
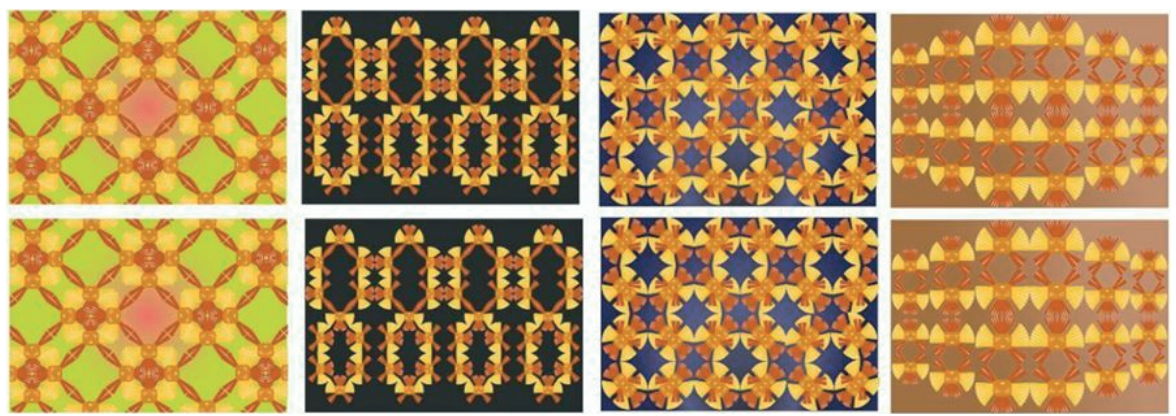

Uso de las memorias técnicas en la elaboración de productos que rescatan la simbología Cañari 
SOMBREROS

TRABAJO DE: Jorge García, Lorena Maldonado, Erika Miranda

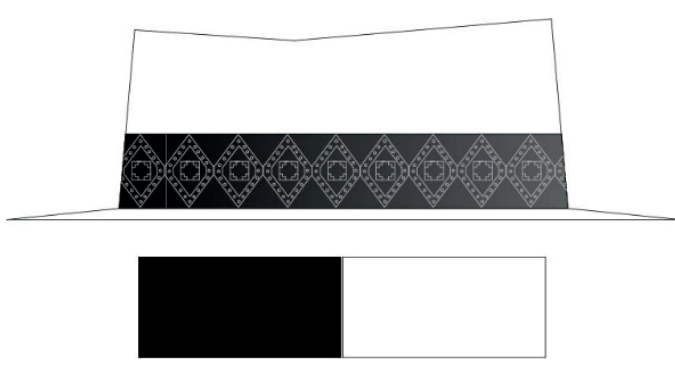

Contraste negro-blanco; representa el día y la noche, la sabiduría y lo desconocido

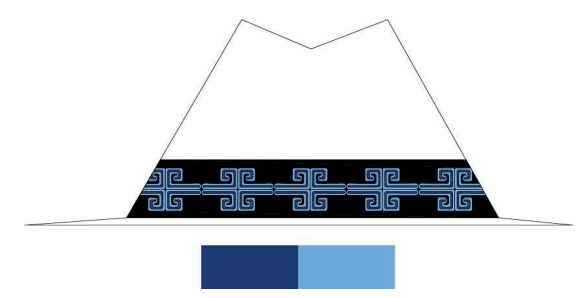

Armonía de azul; refleja el cielo, ríos y lago

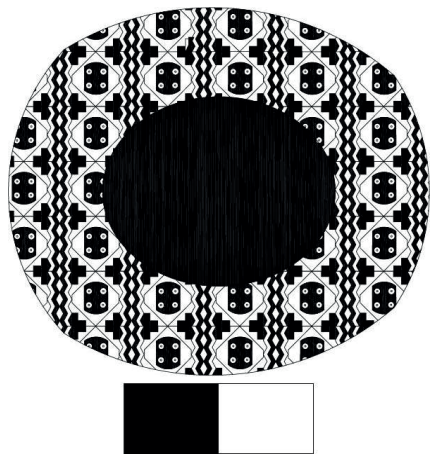

Contraste negro-blanco; representa el día y la noche, la sabiduría y lo desconocido

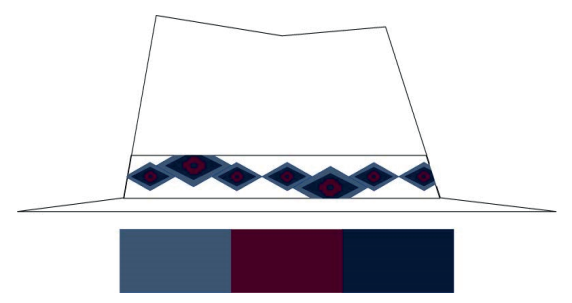

Armonía de azul violáceo contrastado con violeta; representa la madurez y lo nuevo
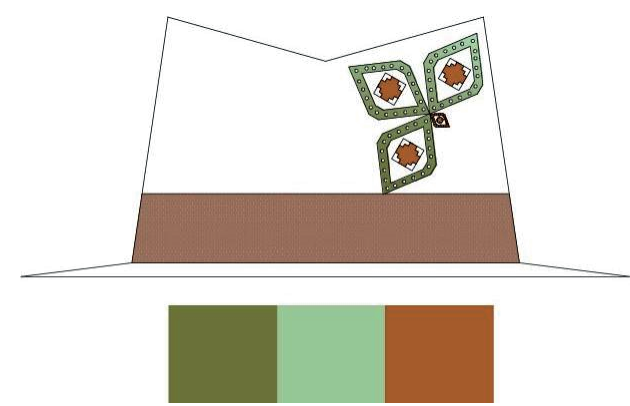

Armonía de verde contrastado con Marrón, verde naturaleza y marrón por los colores tierra.

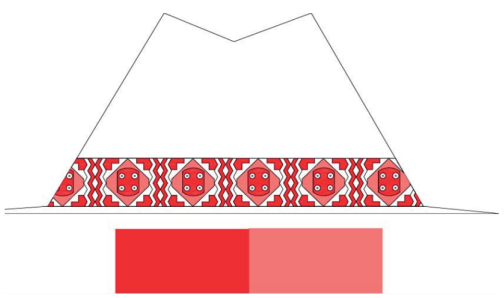

Armonía de rojo; refleja la vida y muerte subjetivamente

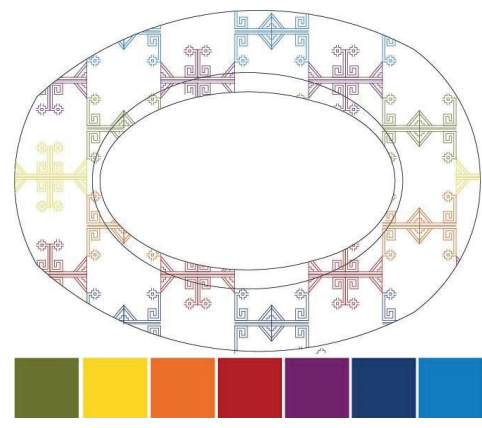

Colores primarios y secundarios; basados en círculo cromático representa colores de la Guacamaya

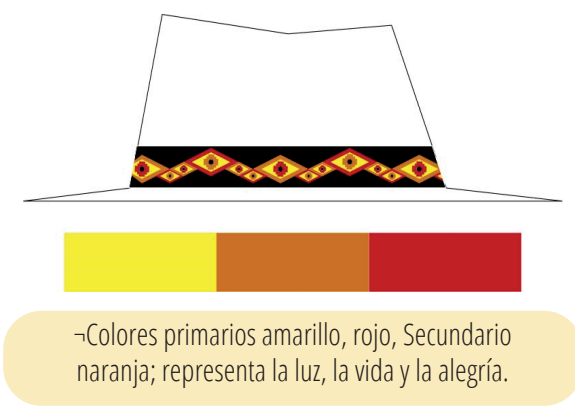




\section{CONVERGENCE TECH}

PLAYERAS

TRABAJO DE: Juan Andrés Proaño Torres, Andrés Sebastián Aguayo Nivelo, Bryan Sebastián Galarza González
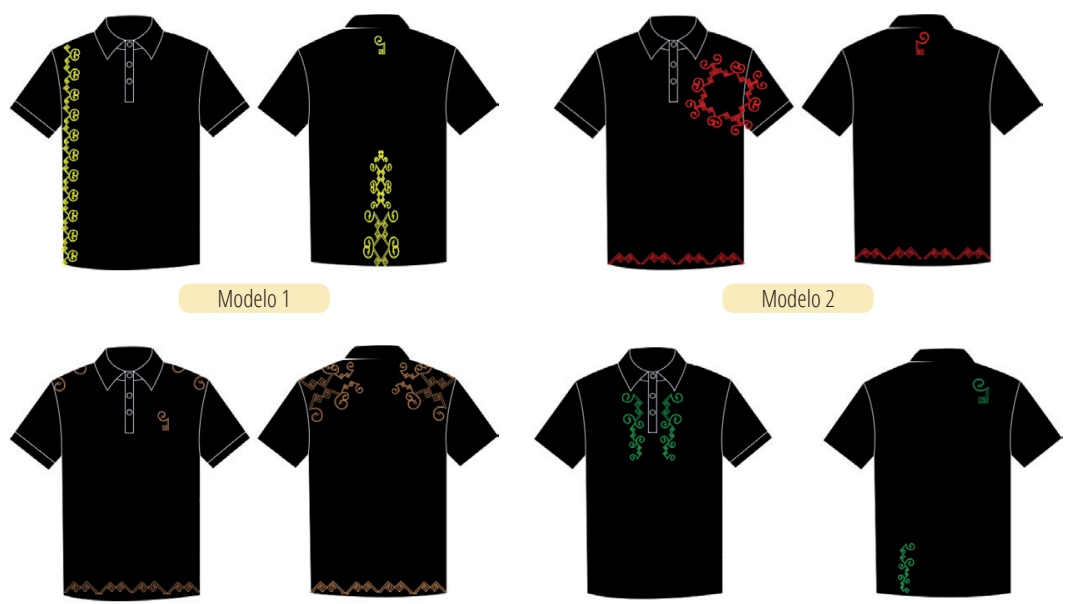

Modelo 3

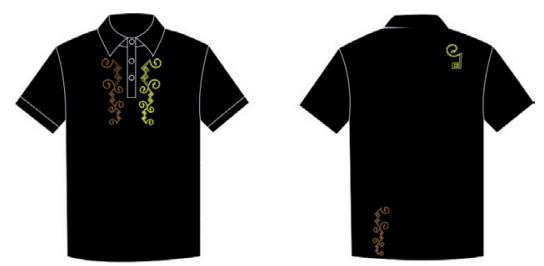

Model0 5

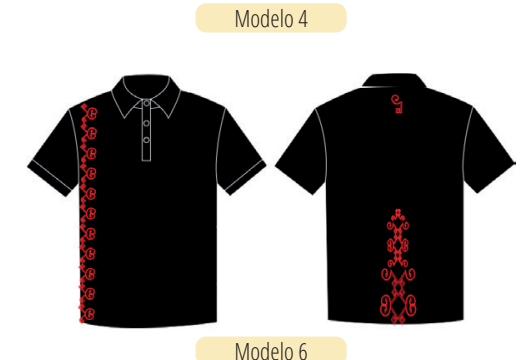

\section{SOMBREROS}

TRABAJO DE: Amoroso Galarza Juan Andrés, Tenezaca Criollo Luis Fernando

Modelos de sombreros a los cuales se les agregó la cintilla, la cual fue trabajada mediante la técnica del sublimado, para rescatar la simbología de la Cultura Cañari. Cintillas para sombreros de paja toquilla.
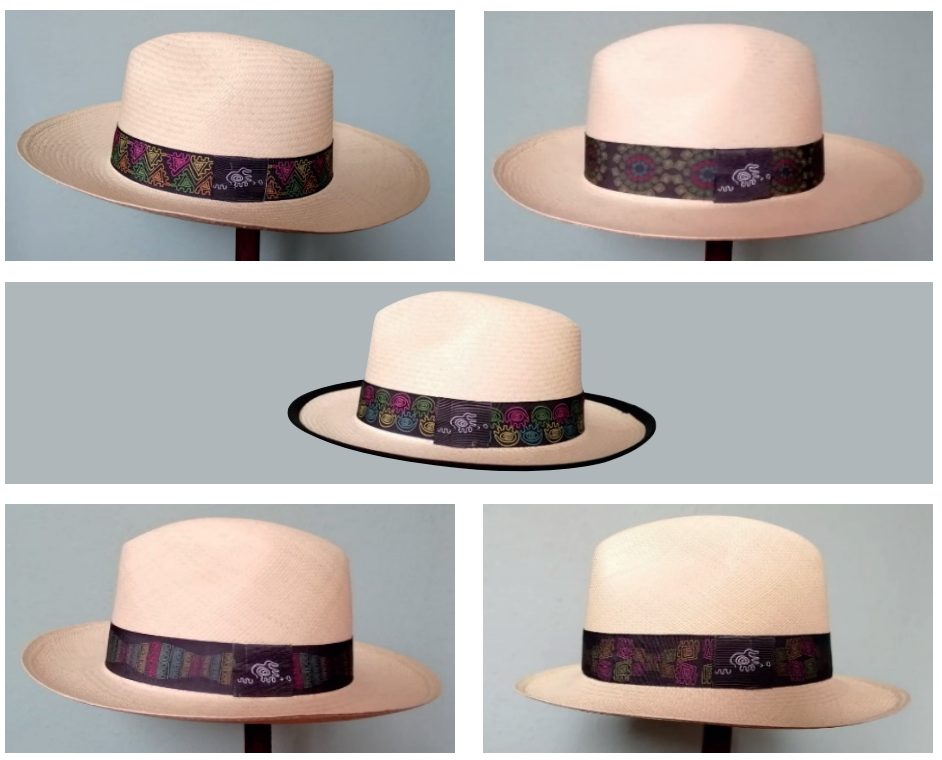

Uso de las memorias técnicas en la elaboración de productos que rescatan la simbología Cañari 


\section{DISCUSIÓN}

$\mathbf{E}$

l cantón Cañar es parte de los siete cantones de la

provincia del mismo nombre. Está ubicado al sur

del Ecuador, al noroeste de la provincia de Cañar.

Limitado al norte por la provincia de Chimborazo; al sur por la provincia del Azuay y los cantones de Biblián y Azogues; al este, por el cantón de Azogues y la oeste, por la provincia del Guayas.

Geográficamente, el cantón Cañar es el más extenso de esta jurisdicción, ocupa el 56.07\% del territorio de la provincia; aunque en los últimos treinta y tres años por su situación de orden político ha tenido un desmembramiento territorial. "El cantón Cañar tiene 59.323 habitantes de los cuales 24.243 son indígenas, equivalente al 38,08\% de la población total". Krainer A y Guerra M. (2012) señala que "Que el porcentaje de indígenas en el cantón Cañar es de 38,08\%" de acuerdo a este autor.

\section{Origen Mitológico}

La palabra "Cañari" se cree que proviene de Ka (culebra) y de Ara (guacamaya). Para algunos académicos el significado es: favorecido de la culebra y guacamaya, considerados como animales importantes de su religión, que de dichos animales se originan leyendas.

\section{Leyenda de las Guacamayas}

Esta leyenda es una de las más conocidas por la mayoría de los pueblos de Cañar.

Según Aguaiza, JP. (2013) “El diluvio Cañar, exterminó a toda la población, menos a dos hermanos que se refugiaron en la montaña más alta llamada Huacayñan o camino de llanto." A continuación, el autor culmina el relato.

Luego de la inundación, mientras las aguas descendían, los dos hermanos salieron a buscar alimentos y a su regreso encontraron en su refugio deliciosos manjares listos y preparados. Dicha situación se repitió por tres días más, sin saber quién les estaba proveyendo de los deliciosos alimentos, así que decidieron que uno se ocultaría, mientras el otro saldría a buscar alimento; estando el mayor al acecho para descubrir el enigma, logro divisar dos guacamayas con cara de mujer, de cabello largo y atado, trató de capturarlas, sin tener éxito los dos primeros días.
Al tercer día, el hermano menor logro atrapar a la guacamaya menor; se casó con ella y tuvo seis hijos, tres varones y tres mujeres, de los cuales descienden los Cañaris. Las guacamayas fueron quienes dieron las semillas a los dos hermanos, para que siembren y cultiven la tierra. Aguaiza, JP. (2018), págs. 25-26.

\section{Leyenda de la Serpiente}

El origen de la serpiente es otra de las leyendas más importantes del origen de los Cañaris, la cual mezcla dos elementos en su narración, la serpiente y la laguna culebrillas.

La leyenda narra, que una enorme serpiente se sumergió en una laguna del territorio Cañari, que luego de dar origen a este pueblo, no volvió a aparecer jamás. Del mismo modo el pueblo Cañari venera las lagunas, siendo lugares sagrados y objeto de culto religioso.

Son tres lagunas consideradas sagradas que guardan una gran profundidad al demiurgo Cañari: las cuales están ubicadas en: Culebrillas ubicada en el Nudo del Azuay, laguna de San Fernando provincia del Azuay y el valle de Yunguilla al sur de la provincia del Azuay. Aguaiza, JP. (2018), pág. 26.

\section{Origen Étnico de los Cañaris}

Este grupo étnico que habita mayormente en Cañar y en un menor porcentaje en Azuay (Sierra sur del Ecuador), que emplean la lengua Kichwa. Su población actualmente se dedica al cultivo de alimentos que crecen en clima frio como: la papa, el melloco, la oca, la quinua, las habas, entre otros.

Nuevos planteamientos sobre la población nativa, gira en torno a desmitificar el pasado de un señorío o jefatura andina, pero que dejo de ser "Cañari" mucho antes de la llegada de los españoles, debido a la coincidencia de los problemas conjuntos, ejemplo, los constantes traslados poblacionales hacia distintos puntos del llamado Tahuantinsuyo, también a las provincias nativas del Ecuador aborigen. Burgos, $\mathrm{H}$ (2003), pág.20.

Los "Régulos practicaron la poligamia, y el primogénito varón tomaba el papel del padre en el cacicazgo. Los jefes no tenían el mismo poder y economía, por dicha carencia formaban alianzas para protegerse contra la opre- 
sión. Esta cultura habita desde las cabeceras del Azuay hasta Saraguro, desde los picos de Gualaquiza hasta las playas de Naranjal y las costas de Jambelí.

En las familias cañaris existieron rasgos particulares; uno de estos fue los Peleusis que se asentaron en la actual ciudad de Azogues.

\section{Idioma Cañari}

Del mismo modo que en otras culturas que se encuentran en el territorio ecuatoriano, la cultura Cañari conserva parte de su dialecto, el Quichua es la lengua que hablan en su mayor parte, el actual pueblo Cañari, no obstante, hemos visto que con el paso del tiempo se habla menos este idioma; son las personas adultas quienes en su mayoría hacen uso de este idioma. Según Burgos, $\mathrm{H}$ (2003), pág. 58. "El Quichua se habla plenamente en la comunidad. El «área nuclear» corresponde históricamente con el conglomerado prehispánico llamado Hatun Cañar. Es bilingüe, pero a nivel familiar y comunal se habla un 80\% de Quichua"

\section{Religión y Creencias.}

Los Cañaris eran politeístas, esto quiere decir que rendían culto a varios dioses. Antes de la conquista incaica, los incas adoraban principalmente a la Luna, a los árboles grandes y las piedras; así también veneraban al sol, osos, pumas, serpientes, guacamayos, monos, ranas, lagunas, cuevas, montañas y a determinados lugares donde se suponía se habían originado sus progenitores. La luna tuvo altares en las cumbres de las montañas, cuando se producían un eclipse, salían al campo con instrumentos musicales para llamar su atención y pedirle que retorne, venciendo a la araña gigante que trata de devorarla.

La medicina natural es parte de la creencia de los Cañaris, pues creen que a través de la medicina natural no se sana solo el dolor físico, sino también se purifica el alma y se alejan los malos espíritus; este tipo de medicina es practicada en ceremonias por el anciano sabio del pueblo, el cual pose conocimientos de la pacha mama "madre tierra" y está en contacto con los astros. Guamán, W. (2016).

\section{Vestimenta de la mujer Cañari}

El atuendo de la mujer Cañari consiste en la pollera, la blusa bordada, la faja, la wallkarina, el tupo (prende- dor), los collares el sombrero. Sin embargo, con el paso de los años han variado los diseños, los colores, el material con el que se confeccionan, e incluso se ha visto influenciada por otras culturas del país.

Polleras: Haciendo una línea de tiempo, las pesadas polleras de bayeta se han dejado de utilizar, dando paso a la bayetilla (tela más delgada), la tela strech, la gamuzilla, los tornasoles y las fibras sintéticas que son las que se utilizan actualmente.

"Las jóvenes ahora llevan las polleras con prensas pequeñitas que son planchadas a máquina, pero hace unos años se planchaba a mano".

Lo que se conserva de las polleras son los bordados en la parte inferior y el color depende "del gusto".

Blusa: Antes las blusas eran únicamente de color blanco con bordados en los codos y en el cuello, pero como se ha innovado en todo, ahora se utilizan blusas de diferentes colores y modelos. Y es que la joven admite que en Cañar hay influencia de las costumbres de otras culturas, sobre todo de Otavalo y de Saraguro.

Wallkarina: La wallkarina que se usaba antes era de bayeta y de color negro, con símbolos como la chakana y el sol, cuyas figuras y textura se creaban con la misma lana, mediante la técnica del amasado, ahora, esta prenda se elabora de tela y esa iconografía de lo sagrado cambió por bordados de flores.

Tupo: Este prendedor, que es parte de la indumentaria cañari, es de plata o de acero, lleva una cinta de color "que no debe faltar", pues según la cosmovisión indígena, es para ahuyentar las malas energías.

Faja: Antiguamente esta prenda se la confeccionaba con lana de borrego en telar de cintura. Aunque la nueva tendencia para las mujeres es utilizar elegantes fajas bordadas que llevan pedrería y un cierre que se ajusta y da forma a la figura femenina.

Sombrero: El sombrero de lana de borrego es lo único que se ha mantenido sin variación de la vestimenta Cañari. Su origen se remonta a la Colonia, se creó para proteger la cabeza del frío y del sol. Tiene dos cintas y dos borlas blancas que representan la dualidad. Naula, P. (2019). 


\section{Vestimenta del hombre Cañari}

Los hombres indígenas utilizan un sombrero hecho de lana de borrego de color blanco, de forma semiesférica faldeada por una pequeña visera que está adornada con una elegante trenza, poncho y pantalón generalmente negro. Guamán, W. (2016), Castillo, L. (2017).

\section{Tradiciones y Fiestas}

Hoy en día el pueblo Cañari, una vez al año celebra una gran fiesta, con el objeto de agradecer a sus dioses por todo lo recibido durante el año. Esta fiesta es conocida como Inti Raymi o fiesta del sol.

El Inti Raymi: Se celebra la última semana de junio, fecha en la cual el pueblo Cañari da gracias a su Dios Sol y de igual manera a la madre tierra por la abundancia recibida durante todo el año, Inti Raymi es un ritual ancestral que los indígenas cañaris reviven mediante ofrendas y rituales enfocados a la purificación de la tierra y del alma de todos los presentes.

En estos rituales que se ofrecen tributos al Dios Sol y a la madre Tierra, también está presente la música tradicional del pueblo, la misma que es interpretada mediante instrumentos de viento, esto con el fin de ponerle ritmo y acompañar a la danza Cañari, cuyos movimientos son parte del ritual de adoración a los dioses. Todas estas ceremonias se las realizan en el complejo arqueológico de Inga pirca y en Cerro Narrio. Guamán, W. (2016).

\section{Actividades}

Artesanía: Los artesanos del pueblo cañari se han ido perdiendo con el pasar del tiempo, y hoy en día son pocos quienes continúan con la elaboración de sus artesanías propias de su cultura, artesanías tales como el poncho, la cushma, las polleras, los rebosos, entre otros elementos que son elaborados con lana de oveja en un telar que es maniobrado por una persona con conocimientos; tal y como se muestra en la imagen.

Los artesanos de hoy en día de la Cultura Cañari, elaboran adornos bordados en las polleras, que son usados principalmente por las mujeres cañaris. Artesanías como estas se sacan a relucir en ocasiones especiales, por ejemplo, en fiestas como el Inti Raymi y el Corpus Cristi. Guamán, W. (2016).
Cerámicas: En la Cultura Cañari tienen dos estilos de cerámicas, correspondientes a dos fases culturales, la Tacalshapa (más antigua, referente al Periodo de Desarrollo Regional- Periodo de Integración), y la segunda Cashaloma (esencia arqueológica de la identidad Cañari). Aguaiza, JP. (2013). pág. 29.

Arqueología: Las piezas arqueológicas cañaris encontradas son una clara evidencia de la importancia que tuvo esta cultura a lo largo de su historia. La mayor parte de sus piezas, están exhibidas en el museo de Guantug (Cañar). Guamán, W. (2016).

Las piezas de barro son pertenecientes a la Cultura de Narrio Cañari, estas piezas arqueológicas fueron encontradas en el Cerro Narrío, y se cree que fueron utilizadas por los cañaris para la preparación y cocción de sus alimentos, y también fueron usadas como adornos ceremoniales.

En Narrío, hay una presencia muy importante de la Cultura Cashaloma especialmente en los vasos campaniformes de base redondeada, los denominados floreros de base anular. Tenecota, D. (2013).

\section{Origen del sombrero de paja toquilla}

Los Panamá hats empiezan su existencia como algo cotidiano y popular, debido a que fueron utilizados por los nativos principalmente de la provincia de Manabí. Aunque estos sombreros eran más grandes y cubrían todo el cuello y las orejas, los colonos quedaron maravillados por los mismos, y a su vez los utilizaron también. Por lo cual se popularizó en la nobleza también y la palma de la cual nacen los tejidos fue bautizada como Carludovica Plamata en honor al rey Carlos IV de España y de su esposa que tenía por nombre Ludovica. Los sombreros de paja toquilla, como los conocemos inician con Manuel Alfaro (padre del ex presidente ecuatoriano Eloy Alfaro), quien inicia un negocio de producción de sombreros, y la exportación de los mismos a Panamá, que ya estaba convirtiéndose en un gran centro de comercio. Cuenca también abre una fábrica de los mismos sombreros, aunque de diferente calidad, posteriormente a esto como un dato encontramos que el Eloy Alfaro llego a exportar 220.000 sombreros de paja toquilla hacia California.

El sombrero de paja toquilla, se populariza a nivel mundial debido a que un comerciante francés que residía en Panamá, llevó los sombreros a una feria mundial, 
que fue realizada en Paris para exhibirlos; al no ser considerado Ecuador, como un país participante los sombreros toman el nombre de Panamá Hats. Incluso se popularizó mucho más en la construcción del canal de Panamá, en donde todos los trabajadores lo utilizaban y hasta el ex presidente de los Estados Unidos, Theodore Roosevelt, fue captado en una fotografía utilizando uno. Así creció tanto su fama que hasta los actores de Hollywood de la época los utilizaban, apareciendo en películas como "Casablanca" y "Lo que el viento se llevó". Palacios, C. (2012).

\section{El proceso de elaboración del sombrero de paja toquilla}

La palma de dónde se extrae la paja toquilla, viene principalmente de la provincia de Manabí, en los sectores de Montecristi, Jipijapa, y San Lorenzo. Es una palmera con hojas en forma de abanico, que necesita de tierra húmeda para crecer, puede ser cultivada durante todo el año y sin mucho cuidado ni mantenimiento. Las hojas son verdes y el centro de las hojas tiene una especie de tallo color marfil, de dónde sale el material para hacer los sombreros.

Al pelar las hojas, el material es trasladado a unos tanques de agua hirviendo, para eliminar el color verdoso del mismo. Luego se debe dejar, secar al aire libre para que, al tejer el material, no se pudra si no está lo suficientemente seco. Al tener el material listo los, artesanos pueden empezar a tejer, con las hormas de madera (normalmente) que les ayuda a formar el sombrero. Antes deben tener listo, el grosor de la paja (el hilo) dependiendo del tejido necesario: tejido 1 grado 0: es 10 pajas en 1 pulgada, grado 1: es 11 pajas en 1 pulgada, grado 2: es 12 pajas en 1 pulgada, etc.

Un sombrero común, podría tomar un día de elaboración de parte del artesano, pero si es un sombrero de mejor calidad, con tejidos más finos podría tomar un tiempo de elaboración de 2 a 6 meses.

Luego del tejido, viene una serie de procesos, para formar perfectamente el sombrero, como: el proceso de asociado, luego lavado, blanqueado, secado, tinturado y prensado. Muchos de estos procesos funcionan con vapor y se utilizan calderos que producen el calor necesario para el blanqueo, lavado, y tinturado. Todos estos procesos se hacen por cada sombrero, y hay muchas personas trabajando detrás de cada paso de elaboración. Palacios, C. (2012).

Por medio de este trabajo se ha determinado la importancia de la simbología y la gama de colores, para rescatar los valores ancestrales de la Cultura Cañari. En el caso de la simbología podemos ver cómo, está plasmada en sus actividades, creencias, religión, vestimentas y fiestas, al implementar un nuevo módulo y plasmarlo en sombreros de paja toquilla por medio del sublimado, se busca adaptar la Cultura Cañari a las tendencias actuales de la moda.

Al ser la Cultura Cañari, muy amplia en historia, creencias y restos arqueológicos nos brinda varias oportunidades, en el capo del diseño, para experimentar mediante el uso de las diversas técnicas del diseño, nuevas formas de expresión de esta cultura, mediante la elaboración de diversos artículos. Se logró plasmar diseños en cintillas, para sombreros de paja toquillas, sin perder el valor y el mensaje que conlleva el símbolo utilizado de la Cultura Cañari, obteniendo una prenda textil representativa del Ecuador.

El proyecto Integrador de Saberes, ha sido una guía, que ha permitido tener ideas y pautas de cómo llevar a cabo la culminación del proyecto.

9. Juan Andrés Proaño Torres

10. Andrés Sebastián Aguayo Nivelo

11. Bryan Sebastián Galarza González

12. Amoroso Galarza Juan Andrés

13. Tenezaca Criollo Luis Fernando

14. Paulina Urgilés

15. Sebastián Arévalo 


\section{BIBLIOGRAFÍA}

Aguaiza, JP. (2013) ANÁLISIS DE LOS RASGOS GRÁFICOS DE LA CULTURA CAÑARI. CREACIÓN DE FAMILIAS TIPOGRÁFICAS. Tesis. Riobamba, Ecuador.

Burgos, H. (2003) LA IDENTIDAD DEL PUEBLO CAÑARI. Abya-Yala. Quito, Ecuador.

Encalada, M. (2018) CO

SMOVISIÓN CAÑARI EN EL DISEÑO DE INDUMENTARIA. Tesis. Universidad del Azuay, Cuenca, Ecuador.

Guamán, W. (2016) CAÑAR: Cultura y tradición. Tesis. Cuenca, Ecuador.

Krainer, A. y Guerra, M. (2012) INTERCULTURALIDAD. UNACERCAMIENTODESDELAINVESTIGACIÓN. FLACSO. Quito, Ecuador.

Naula, P. (2019) El Tiempo: La vestimenta cañari cambia a lo largo de la historia.

Palacios, C. (2012) Los sombreros de paja toquilla un producto artesanal. USFQ. Tesis. Universidad San Francisco de Quito. Quito, Ecuador.

Tenecota, D. (2013) ESTUDIO DE LOS SIGNOS Y SÍMBOLOSDE LA CULTURA CAÑARI, APLICADO AL DISEÑO DE MOBILIARIO PARA UN ESPACIO HABITABLE. Tesis, Cuenca, Ecuador.

Zaruma, V. (2006) Wakanmay, Aliento Sagrado: Perspectivas de Teología India: Una Propuesta Desde la Cultura Cañari. Abya-Yala. Quito, Ecuador. 